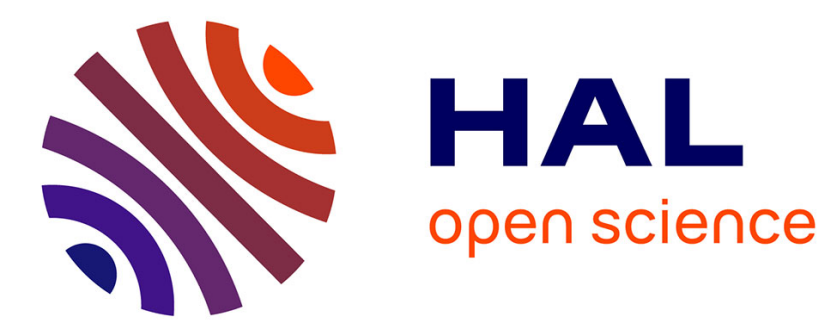

\title{
Simultaneous Energy and Information Transmission in Gaussian Multiple Access Channels
}

\author{
Selma Belhadj Amor, Samir M. Perlaza, Ioannis Krikidis
}

\section{To cite this version:}

Selma Belhadj Amor, Samir M. Perlaza, Ioannis Krikidis. Simultaneous Energy and Information Transmission in Gaussian Multiple Access Channels. The Fifth International Conference on Communications and Networking (ComNet'2015), Nov 2015, Hammamet, Tunisia. hal-01221340

\section{HAL Id: hal-01221340 \\ https://hal.science/hal-01221340}

Submitted on 29 Oct 2015

HAL is a multi-disciplinary open access archive for the deposit and dissemination of scientific research documents, whether they are published or not. The documents may come from teaching and research institutions in France or abroad, or from public or private research centers.
L'archive ouverte pluridisciplinaire HAL, est destinée au dépôt et à la diffusion de documents scientifiques de niveau recherche, publiés ou non, émanant des établissements d'enseignement et de recherche français ou étrangers, des laboratoires publics ou privés. 


\title{
Simultaneous Energy and Information Transmission in Gaussian Multiple Access Channels
}

\author{
Selma Belhadj Amor, Samir M. Perlaza, and Ioannis Krikidis
}

\begin{abstract}
In this paper, simultaneous wireless information and energy transmission is studied from an information theoretic standpoint. The main contribution is twofold: $(i)$ the capacityenergy region of the memoryless Gaussian multiple access channel is fully characterized; and $(i i)$ the maximum sum-rate that can be achieved when a minimum energy level is required at the input of the receiver is determined. In particular, it is shown that the sum-capacity for a given energy threshold can be achieved by a simple power-splitting scheme.
\end{abstract}

Index Terms-RF energy harvesting, capacity-energy region, multiple access channel, Gaussian channels, simultaneous wireless information and energy transfer (SWIET).

\section{INTRODUCTION}

$\mathbf{S}$ IMULTANEOUS wireless information and energy transmission with co-located information and energy receivers is a new communication paradigm, in which receivers extract both information and energy from the same signal [1]. It is a promising technology for low-power short-range communication systems such as sensor networks, machine to machine networks, body area networks, etc. From an information theoretic standpoint, this new paradigm is characterized by a fundamental trade-off between information and energy transmission, since both objectives are often antagonistic [10]. Most of the current work in this area studies this trade-off from a signal-processing or networking point of view [1] and focuses on the feasibility aspects [9]. However, the fundamental limits of this technology are still unknown and only a few works appear in the literature.

The trade-off between information and energy transmission for a basic point-to-point communication was introduced in [10]. This work was extended in [7] for a parallel pointto-point channel (frequency-selective channel). More complex network structures in which decoders are characterized by an additional energy constraint were studied in [5], [6] and [8]. Such additional constraints change the overall optimal performance of the network. In the literature, different forms of received energy constraints were considered in multiuser/multi-hop networks. For instance, Gurakan et al. [8] studied multi-hop networks in which energy is transmitted via a dedicated channel. Gastpar [6] considered the Gaussian mutiaccess channel (G-MAC) under maximum received energy constraint. Fouladgar et al. [5] characterized the capacityenergy region of the two-user discrete memoryless multiaccess channel (MAC) which involves an auxiliary timesharing random variable (RV) $Q$ of cardinality $|Q| \leqslant 4$.

Selma Belhadj Amor and Samir M. Perlaza are with the Institut National de Recherche en Informatique et en Automatique (INRIA), Lyon, France (e-mail: \{selma.belhadj-amor, samir.perlaza\}@inria.fr).

Ioannis Krikidis is with the Dept. of Electrical and Computer Engineering at the University of Cyprus. Nicosia, Cyprus (e-mail: krikidis@ucy.ac.cy).

This research is supported in part by the European Commission under Marie Skłodowska-Curie Individual Fellowship No. 659316.
In the standard G-MAC (without energy constraint), the capacity region was determined independently by Cover [2] and Wyner [11] and it is well-known that time-sharing is not needed $(|Q|=1)$. However, when a minimum energy level is required at the input of the receiver, Fouladgar et al. [5] provided an example of coding scheme for the GMAC that requires additional transmitter coordination through time-sharing in order to satisfy the energy constraint. Consequently, a straightforward extension of the capacity results in the standard G-MAC to the setup with minimum received energy constraints is not possible as it involves considering all possible time-sharing RVs.

This paper studies the G-MAC with a minimum received energy rate constraint and provides an explicit expression of the capacity-energy region. This region is achieved by a simple power-splitting scheme at both transmitters. The maximum sum-rate that can be achieved for a given energy rate required at the input of the receiver is also provided. For clarity of exposition, in what follows only the case of two users is discussed. However, all our results easily extend to arbitrary $K>2$ number of users and for non-colocated receiver and energy-harvester.

\section{Gaussian Multiple Access Channel}

Consider the two-user memoryless G-MAC in Fig. 1. At each channel use $t \in \mathbb{N}, X_{1, t}$ and $X_{2, t}$ denote the real symbols sent by transmitters 1 and 2 , respectively. The receiver observes the real channel output

$$
Y_{t}=h_{1} X_{1, t}+h_{2} X_{2, t}+Z_{t}
$$

while the energy harvester observes

$$
S_{t}=h_{1} X_{1, t}+h_{2} X_{2, t}+Q_{t},
$$

where $h_{1}$ and $h_{2}$ are the corresponding constant non-negative channel coefficients, $Z_{t}$ and $Q_{t}$ are identically distributed zeromean unit-variance real Gaussian random variables. For the ease of explanation, the receiver and the energy harvester are assumed to be co-located, which justifies that they observe the same channel coefficients.

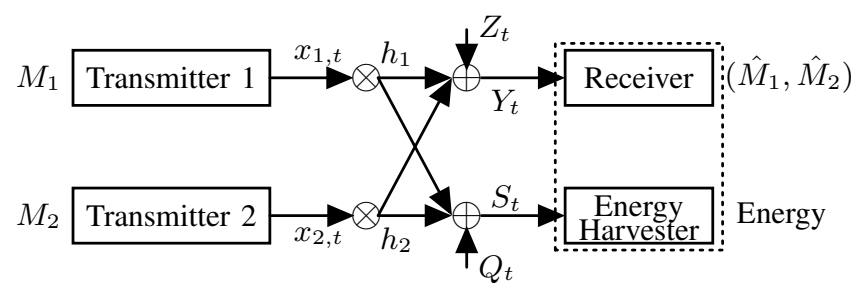

Fig. 1. Two-user memoryless Gaussian MAC. 
The G-MAC introduced above is fully described by two parameters: the signal to noise ratios (SNRs) $\mathrm{SNR}_{1}$ and $\mathrm{SNR}_{2}$, which are defined as follows:

$$
\mathrm{SNR}_{i} \triangleq h_{i}^{2} P_{i}, \quad i \in\{1,2\} .
$$

In this context, two main tasks are to be simultaneously accomplished: information and energy transmission.

\section{A. Information Transmission}

The goal of the communication is to convey the independent messages $M_{1}$ and $M_{2}$ from transmitters 1 and 2 to the common receiver. The messages $M_{1}$ and $M_{2}$ are independent of the noise terms $Z_{1}, \ldots, Z_{N}$ and are uniformly distributed over the sets $\mathcal{M}_{1} \triangleq\left\{1, \ldots,\left\lfloor 2^{n R_{1}}\right\rfloor\right\}$ and $\mathcal{M}_{2} \triangleq\left\{1, \ldots,\left\lfloor 2^{n R_{2}}\right\rfloor\right\}$, where $R_{1}$ and $R_{2}$ denote the transmission rates and $n \in \mathbb{N}$ is the blocklength. The $t$-th symbol of transmitter $i$ depends on its message index $M_{i}$. More specifically,

$$
X_{i, t}=f_{i, t}^{(n)}\left(M_{i}\right), \quad t \in\{1, \ldots, n\},
$$

for some encoding functions

$$
f_{i, t}^{(n)}: \mathcal{M}_{i} \rightarrow \mathbb{R}
$$

The symbols $X_{i, 1}, \ldots, X_{i, n}$ satisfy an expected average input power constraint

$$
\frac{1}{n} \sum_{t=1}^{n} \mathrm{E}\left[X_{i, t}^{2}\right] \leqslant P_{i}
$$

where $P_{i}$ denotes the average transmit power of transmitter $i$ in Joules per channel use, with $i \in\{1,2\}$. The receiver produces an estimation $\left(\hat{M}_{1}^{(n)}, \hat{M}_{2}^{(n)}\right)=\Phi^{(n)}\left(Y^{n}\right)$ of the message-pair $\left(M_{1}, M_{2}\right)$ via a decoding function $\Phi^{(n)}: \mathbb{R}^{n} \rightarrow \mathcal{M}_{1} \times \mathcal{M}_{2}$, and the average probability of error is

$$
P_{\text {error }}^{(n)} \triangleq \operatorname{Pr}\left\{\left(\hat{M}_{1}^{(n)}, \hat{M}_{2}^{(n)}\right) \neq\left(M_{1}, M_{2}\right)\right\} .
$$

\section{B. Energy Transmission}

The energy transmission rate at the energy harvester is

$$
B^{(n)} \triangleq \frac{1}{n} \sum_{t=1}^{n} \mathrm{E}\left[S_{t}^{2}\right] \leqslant 1+\mathrm{SNR}_{1}+\mathrm{SNR}_{2}+2 \sqrt{\mathrm{SNR}_{1} \mathrm{SNR}_{2}} .
$$

The goal of the energy transmission is to guarantee that the energy rate $B^{(n)}$ is not less than a given constant denoted by

$$
0<B \leqslant 1+\mathrm{SNR}_{1}+\mathrm{SNR}_{2}+2 \sqrt{\mathrm{SNR}_{1} \mathrm{SNR}_{2}} .
$$

Hence, the probability of energy outage is defined as follows

$$
P_{\text {outage }}^{(n)}(\epsilon)=\operatorname{Pr}\left\{B^{(n)}<B-\epsilon\right\},
$$

for some $\epsilon>0$ arbitrarily small.

\section{Simultaneous Information and Energy Transmission}

The MAC in Fig. 1 operates at the information-energy rate triplet $\left(R_{1}, R_{2}, B\right) \in \mathbb{R}_{+}^{3}$ if both transmitters and the receiver use a transmit-receive configuration such that: $(a)$ reliable communication at rates $R_{1}$ and $R_{2}$ is ensured; and $(b)$ the energy transmission rate during the whole block-length $n$ is higher than $B$. Under these conditions, the information-energy rate triplet $\left(R_{1}, R_{2}, B\right)$ is said to be achievable.

Definition 1 (Achievable Information-Energy Rate Triplets $\left.\left(R_{1}, R_{2}, B\right)\right)$. The triplet $\left(R_{1}, R_{2}, B\right) \in \mathbb{R}_{+}^{3}$ is achievable if there exists a sequence of encoding and decoding functions $\left\{\left\{f_{1, t}^{(n)}\right\}_{t=1}^{n},\left\{f_{2, t}^{(n)}\right\}_{t=1}^{n}, \Phi^{(n)}\right\}_{n=1}^{\infty}$ such that both the average error probability and the energy-outage probability tend to zero as the blocklength $n$ tends to infinity. That is,

$$
\begin{aligned}
& \limsup _{n \rightarrow \infty} P_{\text {error }}^{(n)} \quad=0, \\
& \limsup _{n \rightarrow \infty} P_{\text {outage }}^{(n)}(\epsilon)=0, \quad \text { for any } \epsilon>0 .
\end{aligned}
$$

The fundamental limits on the information-energy trade-off are accurately modeled by the capacity-energy region.

Definition 2 (Capacity-Energy Region). The capacity-energy region $\mathcal{E}\left(\mathrm{SNR}_{1}, \mathrm{SNR}_{2}\right)$ of the $G-M A C$ is the closure of all achievable information-energy rate triplets $\left(R_{1}, R_{2}, B\right)$.

\section{Main Results}

\section{A. Capacity-Energy Region}

The capacity-energy region of the G-MAC is fully characterized by the following theorem.

Theorem 1 (Capacity-Energy Region). The capacity-energy region $\mathcal{E}\left(\mathrm{SNR}_{1}, \mathrm{SNR}_{2}\right)$ of the $G-M A C$ is the set of all nonnegative information-energy rate triplets $\left(R_{1}, R_{2}, B\right)$ that satisfy

$$
\begin{aligned}
R_{1} \quad & \leqslant \frac{1}{2} \log _{2}\left(1+\beta_{1} \mathrm{SNR}_{1}\right), \\
R_{2} \leqslant & \frac{1}{2} \log _{2}\left(1+\beta_{2} \mathrm{SNR}_{2}\right), \\
R_{1}+R_{2} \leqslant & \frac{1}{2} \log _{2}\left(1+\beta_{1} \mathrm{SNR}_{1}+\beta_{2} \mathrm{SNR}_{2}\right), \\
B \quad \leqslant 1+\mathrm{SNR}_{1}+\mathrm{SNR}_{2} & +2 \sqrt{\left(1-\beta_{1}\right) \mathrm{SNR}_{1}\left(1-\beta_{2}\right) \mathrm{SNR}_{2},}
\end{aligned}
$$

with $\left(\beta_{1}, \beta_{2}\right) \in[0,1]^{2}$.

The proof of Theorem 1 is presented in Section IV. The remaining of this section highlights some important intuitions arising from Theorem 1.

Fig. 2 shows the capacity-energy region $\mathcal{E}\left(\mathrm{SNR}_{1}, \mathrm{SNR}_{2}\right)$ when $\mathrm{SNR}_{1}=\mathrm{SNR}_{2}=10$ and illustrates the three possible information-energy transmission regimes depending on the required minimum energy transmission rate $B:$ (i) $B \in\left[0,1+\mathrm{SNR}_{1}+\mathrm{SNR}_{2}\right] ; \quad$ (ii) $B \in$ $\left(1+\mathrm{SNR}_{1}+\mathrm{SNR}_{2}, 1+\mathrm{SNR}_{1}+\mathrm{SNR}_{2}+2 \sqrt{\mathrm{SNR}_{1} \mathrm{SNR}_{2}}\right] ;$ and (iii) $B \in\left(1+\mathrm{SNR}_{1}+\mathrm{SNR}_{2}+2 \sqrt{\mathrm{SNR}_{1} \mathrm{SNR}_{2}}, \infty\right)$.

In Theorem 1, the terms $\beta_{1}$ and $\beta_{2}$ might be interpreted as the fractions of power that transmitters 1 and 2, respectively, allocate for information transmission. Following this interpretation, it holds that for any achievable triplet $\left(R_{1}, R_{2}, B\right)$, 

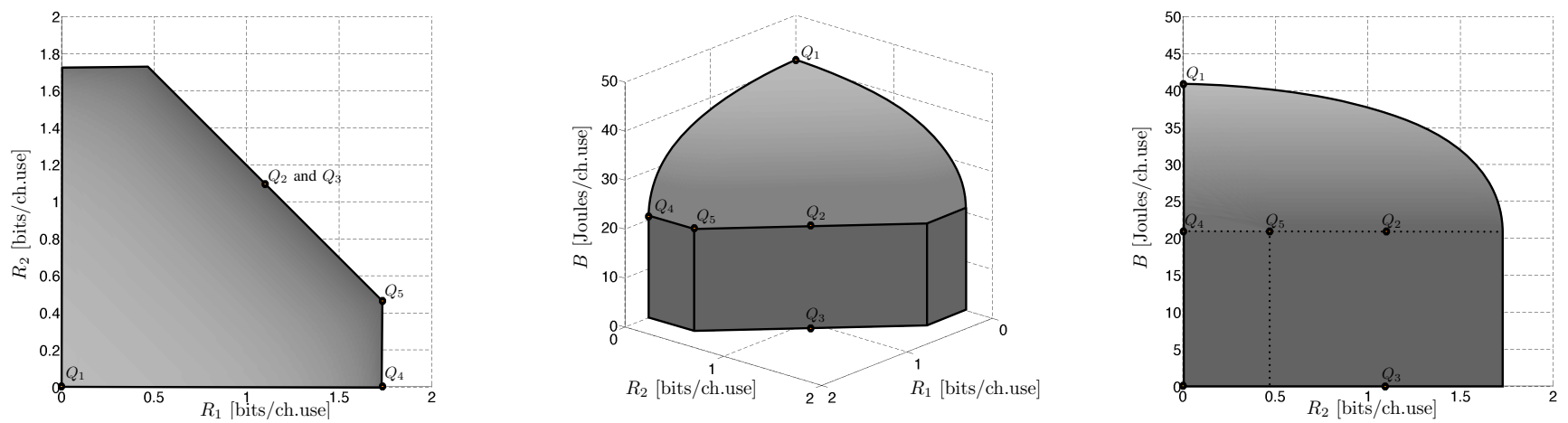

Fig. 2. 3-D representation of the capacity-energy region $\mathcal{E}(10,10)$ in the plane $\left(R_{1}, R_{2}, B\right)$. Left and right figures represent a bi-dimensional view in the $R_{1}-R_{2}$ and $B-R_{2}$ planes of $\mathcal{E}(10,10)$, respectively. The figure in the center is a 3 -D representation of $\mathcal{E}(10,10)$. Note that poins $Q_{1}, Q_{2}$, and $Q_{3}$ are coplanar and satisfy that $R_{1}=R_{2}$. In particular, $Q_{1}=\left(0,0,1+\mathrm{SNR}_{1}+\mathrm{SNR}_{2}+2 \sqrt{\mathrm{SNR}_{1} \mathrm{SNR}_{2}}\right)$; and the points $Q_{2}$ and $Q_{3}$ are also collinear and $R_{1}=R_{2}=\frac{1}{4} \log _{2}\left(1+\mathrm{SNR}_{1}+\mathrm{SNR}_{2}\right)$. The points $Q_{2}, Q_{4}$ and $Q_{5}$ are coplanar and they satisfy $B=1+\mathrm{SNR}_{1}+\mathrm{SNR}_{2}$. In particular, $Q_{4}=\left(\frac{1}{2} \log _{2}\left(1+\mathrm{SNR}_{1}\right), 0,1+\mathrm{SNR}_{1}+\mathrm{SNR}_{2}\right)$ and $Q_{5}=\left(\frac{1}{2} \log _{2}\left(1+\mathrm{SNR}_{1}\right), \frac{1}{2} \log _{2}\left(1+\frac{\mathrm{SNR}_{1}}{1+\mathrm{SNR}_{2}}\right), 1+\mathrm{SNR}_{1}+\mathrm{SNR}_{2}\right)$.

whenever the required minimum energy rate $B$ is smaller than the minimum energy rate required to guarantee reliable communications at rates $R_{1}$ and $R_{2}$, the energy constraint is vacuous. This is basically because transmitting information is always enough to satisfy the energy rate constraint, which implies that $\beta_{1}=\beta_{2}=1$.

Note that any intersection of the volume $\mathcal{E}\left(\mathrm{SNR}_{1}, \mathrm{SNR}_{2}\right)$ with a plane $B=b$, with $b \in\left[0,1+\mathrm{SNR}_{1}+\mathrm{SNR}_{2}\right]$, corresponds to the set of triplets $\left(R_{1}, R_{2}, b\right)$, in which $\left(R_{1}, R_{2}\right) \in$ $\mathcal{C}\left(\mathrm{SNR}_{1}, \mathrm{SNR}_{2}\right)$, with $\mathcal{C}\left(\mathrm{SNR}_{1}, \mathrm{SNR}_{2}\right)$ the capacity region of the G-MAC with parameters $\mathrm{SNR}_{1}$ and $\mathrm{SNR}_{2}$. That is, when the energy rate constraint consists in guaranteeing an energy rate that is lower than the maximum rate achievable with independent symbols, all information rates in $\mathcal{C}\left(\mathrm{SNR}_{1}, \mathrm{SNR}_{2}\right)$ are achievable. (See for instance points $Q_{2}, Q_{3}, Q_{4}$, and $Q_{5}$ in Fig. 2.)

On the other hand, when the energy rate constraint consists in guaranteeing an energy rate $B$ Joules per channel use, with $B \in\left(1+\mathrm{SNR}_{1}+\mathrm{SNR}_{2}, 1+\mathrm{SNR}_{1}+\mathrm{SNR}_{2}+2 \sqrt{\mathrm{SNR}_{1} \mathrm{SNR}_{2}}\right]$, that is, when the energy rate $B$ at the input of the receiver is required to be higher than what is strictly necessary to guarantee reliable communication, transmitters deal with a trade-off between information and energy transmission through powersplitting, i.e., $0 \leqslant \beta_{i}<1$, for $i \in\{1,2\}$.

Finally, requiring a received energy level $B$ within the interval $\left(1+\mathrm{SNR}_{1}+\mathrm{SNR}_{2}+2 \sqrt{\mathrm{SNR}_{1} \mathrm{SNR}_{2}}, \infty\right)$ constraints the achievability of any positive rate (See point $Q_{1}$ in Fig. 2, which is achievable with $\left(\beta_{1}=\beta_{2}=0\right)$ ).

\section{B. Sum-Capacity with Minimum Energy Rate Constraint}

The maximum information sum-rate $R_{\text {sum }}\left(\mathrm{SNR}_{1}, \mathrm{SNR}_{2}, B\right)$ of the G-MAC with parameters $\mathrm{SNR}_{1}$ and $\mathrm{SNR}_{2}$ subject to a minimum energy rate constraint $B$ is the solution to an optimization problem of the form:

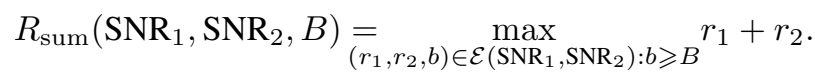

The solution to this problem is given by the following theorem.

Theorem 2 (Sum-Capacity with Minimum Energy Rate). The information sum-capacity of the G-MAC with minimum received energy rate $B$ Joules per channel use is:

$$
R_{\mathrm{sum}}\left(\mathrm{SNR}_{1}, \mathrm{SNR}_{2}, B\right)=\frac{1}{2} \log _{2}\left(1+\beta^{*}\left(\mathrm{SNR}_{1}+\mathrm{SNR}_{2}\right)\right)
$$

with $\beta^{*}=\min \left\{1,\left(\frac{1+\mathrm{SNR}_{1}+\mathrm{SNR}_{2}+2 \sqrt{\mathrm{SNR}_{1} \mathrm{SNR}_{2}}-B}{2 \sqrt{\mathrm{SNR}_{1} \mathrm{SNR}_{2}}}\right)^{+}\right\}$.

Proof: The sum-rate maximization problem in (14) can be written as:

$$
\begin{gathered}
R_{\text {sum }}\left(\mathrm{SNR}_{1}, \mathrm{SNR}_{2}, B\right)=\max _{\left(\beta_{1}, \beta_{2}\right) \in[0,1]^{2}} f_{0}\left(\beta_{1}, \beta_{2}\right) \\
\text { subject to: } \quad g_{0}\left(\beta_{1}, \beta_{2}\right) \geqslant B,
\end{gathered}
$$

where the functions $f_{0}$ and $g_{0}$ are defined as

$$
\begin{array}{r}
f_{0}\left(\beta_{1}, \beta_{2}\right) \triangleq \min \left\{\frac{1}{2} \log _{2}\left(1+\beta_{1} \mathrm{SNR}_{1}+\beta_{2} \mathrm{SNR}_{2}\right),\right. \\
\left.\frac{1}{2} \log _{2}\left(1+\beta_{1} \mathrm{SNR}_{1}\right)+\frac{1}{2} \log _{2}\left(1+\beta_{2} \mathrm{SNR}_{2}\right)\right\}
\end{array}
$$

and

$$
\begin{aligned}
g_{0}\left(\beta_{1}, \beta_{2}\right) & \triangleq 1+\mathrm{SNR}_{1}+\mathrm{SNR}_{2} \\
& +2 \sqrt{\left(1-\beta_{1}\right) \mathrm{SNR}_{1}\left(1-\beta_{2}\right) \mathrm{SNR}_{2} .}
\end{aligned}
$$

For any nonnegative $\beta_{1}$ and $\beta_{2}$ it can be shown that

$$
f_{0}\left(\beta_{1}, \beta_{2}\right)=\frac{1}{2} \log _{2}\left(1+\beta_{1} \mathrm{SNR}_{1}+\beta_{2} \mathrm{SNR}_{2}\right),
$$

and thus the function $f_{0}$ is monotonically increasing in $\left(\beta_{1}, \beta_{2}\right)$. The function $g_{0}$ is motonically decreasing in $\left(\beta_{1}, \beta_{2}\right)$.

Note that for any $\left(\beta_{1}, \beta_{2}\right) \in[0,1]^{2}$, it follows that

$$
g_{0}\left(\min \left(\beta_{1}, \beta_{2}\right), \min \left(\beta_{1}, \beta_{2}\right)\right) \geqslant g_{0}\left(\beta_{1}, \beta_{2}\right)
$$

and

$$
\begin{aligned}
& f_{0}\left(\min \left(\beta_{1}, \beta_{2}\right), \min \left(\beta_{1}, \beta_{2}\right)\right) \leqslant f_{0}\left(\beta_{1}, \beta_{2}\right) \\
& \leqslant f_{0}\left(\max \left(\beta_{1}, \beta_{2}\right), \max \left(\beta_{1}, \beta_{2}\right)\right)
\end{aligned}
$$

with equality in (20) and (21) only when $\beta_{1}=\beta_{2}$. Consequently, the sum-rate maximization problem (16) can also be written as:

$$
\begin{aligned}
& R_{\text {sum }}\left(\mathrm{SNR}_{1}, \mathrm{SNR}_{2}, B\right)=\max _{\beta \in[0,1]} f_{0}(\beta, \beta) \\
& \text { subject to: } \quad g_{0}(\beta, \beta) \geqslant B .
\end{aligned}
$$

This implies that the optimal $\beta^{*}$ is the highest feasible value:

$$
\beta^{*}=\min \left\{1,\left(\frac{1+\mathrm{SNR}_{1}+\mathrm{SNR}_{2}+2 \sqrt{\mathrm{SNR}_{1} \mathrm{SNR}_{2}}-B}{2 \sqrt{\mathrm{SNR}_{1} \mathrm{SNR}_{2}}}\right)^{+}\right\},
$$


which implies that $R_{\text {sum }}\left(\mathrm{SNR}_{1}, \mathrm{SNR}_{2}, B\right)=$ $\frac{1}{2} \log _{2}\left(1+\beta^{*}\left(\mathbf{S N R}_{1}+\mathrm{SNR}_{2}\right)\right)$ and completes the proof.

Note that, if $0 \leqslant B \leqslant 1+\mathrm{SNR}_{1}+\mathrm{SNR}_{2}$, then $\beta^{*}=1$ and all the available power can be dedicated to the information transmission task. Alternatively, if $1+\mathrm{SNR}_{1}+$ $\mathrm{SNR}_{2}<B \leqslant 1+\mathrm{SNR}_{1}+\mathrm{SNR}_{2}+2 \sqrt{\mathrm{SNR}_{1} \mathrm{SNR}_{2}}$, then $\beta^{*}=\frac{1+\mathrm{SNR}_{1}+\mathrm{SNR}_{2}+2 \sqrt{\mathrm{SNR}_{1} \mathrm{SNR}_{2}}-B}{2 \sqrt{\mathrm{SNR}_{1} \mathrm{SNR}_{2}}}$ and there is a strict tradeoff between information and energy transmissions. Finally, if $B>1+\mathrm{SNR}_{1}+\mathrm{SNR}_{2}+2 \sqrt{\mathrm{SNR}_{1} \mathrm{SNR}_{2}}$, then $\beta^{*}=0$ and all the available power must be used for energy transmission.

Remark 1. Time-sharing with power-control suggested in Fouladgar et al.'s example [5] is strictly suboptimal and does not achieve sum-capacity for a given minimum received energy constraint.

Consider the sum-rate optimization problem proposed in [5] in which both users use time-sharing with power control. Specifically, a time-sharing parameter $\lambda \in[0,1]$ is chosen and each transmitter $i$ chooses powers $P_{i}^{\prime}$ (power dedicated to information transmission) and $P_{i}^{\prime \prime}$ (power dedicated to energy transmission). Then, the sum-rate maximization problem proposed in [5] can be written as

$\max _{\left(\lambda, P_{1}^{\prime}, P_{1}^{\prime \prime}, P_{2}^{\prime}, P_{2}^{\prime \prime}\right) \in[0,1] \times \mathbb{R}_{+}^{4}} \frac{\lambda}{2} \log _{2}\left(1+h_{1}^{2} P_{1}^{\prime}+h_{2}^{2} P_{2}^{\prime}\right)$

subject to : $\quad \lambda P_{i}^{\prime}+(1-\lambda) P_{i}^{\prime \prime} \leqslant P_{i}, \quad i \in\{1,2\}$

$1+\lambda\left(h_{1}^{2} P_{1}^{\prime}+h_{2}^{2} P_{2}^{\prime}\right)+(1-\lambda)\left(h_{1} \sqrt{P_{1}^{\prime \prime}}+h_{2} \sqrt{P_{2}^{\prime \prime}}\right)^{2} \geqslant B$.

For any feasible choice of $\left(\lambda, P_{1}^{\prime}, P_{1}^{\prime \prime}, P_{2}^{\prime}, P_{2}^{\prime \prime}\right)$, by the concavity of the logarithm, it follows that

$\frac{\lambda}{2} \log _{2}\left(1+h_{1}^{2} P_{1}^{\prime}+h_{2}^{2} P_{2}^{\prime}\right) \leqslant \frac{1}{2} \log _{2}\left(1+\lambda\left(h_{1}^{2} P_{1}^{\prime}+h_{2}^{2} P_{2}^{\prime}\right)\right)$.

Now, when $\lambda=1$, then (24) holds with equality and the problem (23) can be written as

$$
\begin{gathered}
\max _{\left(P_{1}^{\prime}, P_{2}^{\prime}\right) \in \mathbb{R}_{+}^{2}} \frac{1}{2} \log _{2}\left(1+h_{1}^{2} P_{1}^{\prime}+h_{2}^{2} P_{2}^{\prime}\right) \\
\text { subject to : } \quad P_{i}^{\prime} \leqslant P_{i}, \quad i \in\{1,2\}, \\
1+h_{1}^{2} P_{1}^{\prime}+h_{2}^{2} P_{2}^{\prime} \geqslant B,
\end{gathered}
$$

which is unfeasible for any $B>1+\mathrm{SNR}_{1}+\mathrm{SNR}_{2}$. When $\lambda \in[0,1)$, then (24) holds with strict inequality and the rate $\frac{1}{2} \log _{2}\left(1+\lambda\left(h_{1}^{2} P_{1}^{\prime}+h_{2}^{2} P_{2}^{\prime}\right)\right)$ is always achievable by a power-splitting scheme in which $\beta_{i}=\lambda \frac{P_{i}^{\prime}}{P_{i}}$, with $i \in\{1,2\}$, for any optimal tuple $\left(\lambda, P_{1}^{\prime}, P_{1}^{\prime \prime}, P_{2}^{\prime}, P_{2}^{\prime \prime}\right)$ in (23). This shows that the maximum-sum rate achieved via time-sharing with power control is always bounded away from the sum-capacity (Theorem 2), except when $\lambda=1$. That is, time-sharing with power control is optimal in terms of sum-rate only when the energy constraint is not active, i.e., $B \in\left[0,1+\mathrm{SNR}_{1}+\mathrm{SNR}_{2}\right]$.

\section{PROOF OF THEOREM 1}

\section{A. Proof of Achievability}

Consider that each transmitter $i$, with $i \in\{1,2\}$, uses a fraction $\beta_{i} \in[0,1]$ of its available power to transmit information and uses the remaining fraction of power $\left(1-\beta_{i}\right)$ to transmit energy. Given a power-split $\left(\beta_{1}, \beta_{2}\right) \in[0,1]^{2}$, the achievability of rate-pairs satisfying (13a)-(13c) follows the scheme described in [2], [11]. Additionnaly, in order to satisfy the received energy constraint (13d), the transmitters send a common randomness that is known to both transmitters and to the receiver using all their remaining power. This common randomness does not carry any information and does not produce any interference over the information-carrying signals. More specifically, at each time $t$, transmitter $i$ 's channel input can be written as:

$$
X_{i, t}=\sqrt{\left(1-\beta_{i}\right) P_{i}} W_{t}+U_{i, t}, \quad i \in\{1,2\},
$$

for some independent zero-mean Gaussian informationcarrying symbols $U_{1, t}$ and $U_{2, t}$ with variances $\beta_{1} P_{1}$ and $\beta_{2} P_{2}$, respectively, and independent thereof $W_{t}$ is a zero-mean unit-variance Gaussian energy-carrying symbol known noncausally to all terminals. That is, the rate-pairs $\left(R_{1}, R_{2}\right)$ can be made arbitrarily close to the upper-bounds in (13a)-(13c) by approximating transmitters 1 and 2 channel input symbols by Gaussian RVs with variances $\beta_{1} P_{1}$ and $\beta_{2} P_{2}$, respectively.

The receiver subtracts first the common randomness and then performs successive decoding to recover the messages $M_{1}$ and $M_{2}$.

In the sequel, only the average received energy of the coding scheme given by $B^{(n)} \triangleq \frac{1}{n} \sum_{t=1}^{n} S_{t}^{2}$ is analyzed.

By the choice of $\left(X_{1, t}, X_{2, t}\right)$, the sequence $S_{1}, \ldots, S_{n}$ is i.i.d. and each $S_{t}$ follows a zero-mean Gaussian distribution with variance $B$ where

$$
\begin{aligned}
B \triangleq 1 & +\mathrm{SNR}_{1}+\mathrm{SNR}_{2} \\
& +2 \sqrt{\left(1-\beta_{1}\right) \mathrm{SNR}_{1}\left(1-\beta_{2}\right) \mathrm{SNR}_{2}} .
\end{aligned}
$$

By the weak law of large numbers, it holds that $\forall \epsilon>0$

$$
\lim _{n \rightarrow \infty} \operatorname{Pr}\left(\left|B^{(n)}-B\right|>\epsilon\right)=0 .
$$

Consequently,

$$
\begin{aligned}
& \lim _{n \rightarrow \infty} \operatorname{Pr}\left(B^{(n)}>B+\epsilon\right)=0, \quad \text { and } \\
& \lim _{n \rightarrow \infty} \operatorname{Pr}\left(B^{(n)}<B-\epsilon\right)=0 .
\end{aligned}
$$

From (29b), it holds that $\forall b \in[0, B]$,

$$
\lim _{n \rightarrow \infty} \operatorname{Pr}\left(B^{(n)}<b-\epsilon\right)=0
$$

which completes the proof of achievability of Theorem 1 .

\section{B. Proof of Converse}

Fix an information-energy rate triplet $\left(R_{1}, R_{2}, B\right) \in$ $\mathcal{E}\left(\mathrm{SNR}_{1}, \mathrm{SNR}_{2}\right)$. For this information-energy rate triplet and for each blocklength $n$, fix encoding and decoding functions such that

$$
\begin{aligned}
\limsup _{n \rightarrow \infty} P_{\text {error }}^{(n)} & =0, \\
\limsup _{n \rightarrow \infty} P_{\text {outage }}^{(n)}(\epsilon) & =0 \text { for any } \epsilon>0,
\end{aligned}
$$

and such that the input power constraint (6) is satisfied for each $n$. 
Using assumption (31a), applying Fano's inequality under the assumption that the common randomness sequence $\left\{W_{t}\right\}$ is known non-causally to all terminals and following similar steps as in [2] and [11], it can be shown that the rates $\left(R_{1}, R_{2}\right)$ must satisfy:

$$
\begin{aligned}
n R_{1} & \leqslant \sum_{t=1}^{n} I\left(X_{1, t} ; Y_{t} \mid X_{2, t}, W_{t}\right)+\epsilon_{1}^{(n)}, \\
n R_{2} & \leqslant \sum_{t=1}^{n} I\left(X_{2, t} ; Y_{t} \mid X_{1, t}, W_{t}\right)+\epsilon_{2}^{(n)}, \\
n\left(R_{1}+R_{2}\right) & \leqslant \sum_{t=1}^{n} I\left(X_{1, t} X_{2, t} ; Y_{t} \mid W_{t}\right)+\epsilon_{12}^{(n)},
\end{aligned}
$$

where $\frac{\epsilon_{1}^{(n)}}{n}, \frac{\epsilon_{2}^{(n)}}{n}$, and $\frac{\epsilon_{1}^{(n)}}{n}$ tend to zero as $n$ tends to infinity.

Using assumption (31b), for a given $\epsilon_{n}>0$, for any $\eta>0$ there exists $n_{0}(\eta)$ such that for any $n \geq n_{0}(\eta)$, it holds

$$
\operatorname{Pr}\left(B^{(n)} \geqslant B-\epsilon_{n}\right) \geqslant 1-\eta
$$

From Markov's inequality [4], the following holds

$$
\left(B-\epsilon_{n}\right) \operatorname{Pr}\left(B^{(n)} \geqslant B-\epsilon_{n}\right) \leqslant \mathrm{E}\left[B^{(n)}\right] .
$$

Combining (33) and (34) yields

$$
\left(B-\epsilon_{n}\right)(1-\eta) \leqslant \mathrm{E}\left[B^{(n)}\right] .
$$

which can be written as

$$
\left(B-\delta_{n}\right) \leqslant \mathrm{E}\left[B^{(n)}\right],
$$

for some $\delta_{n}>\epsilon_{n}$ (for a sufficiently large $n$ ).

The following evaluates the bounds in (32) and (36) for the G-MAC.

Here we assume that at each time $t$ the channel input $X_{i, t}=U_{i, t}+\alpha_{i, t} W_{t}$ where $U_{i, t}$ a zero-mean informationcarrying component with variance $\sigma_{i, t}^{2}$, independent thereof $W_{t}$ is a zero-mean unit-variance energy-carrying component that is known to all terminals, and the coefficients $\alpha_{i, t} \in \mathbb{R}$ are deterministic and known to all terminals.

Define $\alpha_{i}^{2} \triangleq \frac{1}{n} \sum_{t=1}^{n} \alpha_{i, t}^{2}$ and $\sigma_{i}^{2} \triangleq \frac{1}{n} \sum_{t=1}^{n} \sigma_{i, t}^{2}$, for $i \in\{1,2\}$.

The input sequence must satisfy the input power constraint (6) which can be written, for $i \in\{1,2\}$, as:

$$
\frac{1}{n} \sum_{t=1}^{n} \mathrm{E}\left[X_{i, t}^{2}\right]=\frac{1}{n} \sum_{t=1}^{n}\left(\sigma_{i, t}^{2}+\alpha_{i, t}^{2}\right)=\sigma_{i}^{2}+\alpha_{i}^{2} \leqslant P_{i} .
$$

For the considered $\left(X_{1, t}, X_{2, t}\right)$ the bounds in (32) can be written as

$$
\begin{aligned}
n R_{1} & \leqslant \sum_{t=1}^{n} \frac{1}{2} \log _{2}\left(1+h_{1}^{2} \sigma_{1, t}^{2}\right)+\epsilon_{1}^{(n)}, \\
n R_{2} & \leqslant \sum_{t=1}^{n} \frac{1}{2} \log _{2}\left(1+h_{2}^{2} \sigma_{2, t}^{2}\right)+\epsilon_{2}^{(n)}, \\
n\left(R_{1}+R_{2}\right) & \leqslant \sum_{t=1}^{n} \frac{1}{2} \log _{2}\left(1+h_{1}^{2} \sigma_{1, t}^{2}+h_{2}^{2} \sigma_{2, t}^{2}\right)+\epsilon_{12}^{(n)},
\end{aligned}
$$

and the average received energy is given by:

$$
\begin{aligned}
\mathrm{E}\left[B^{(n)}\right]= & h_{1}^{2}\left(\frac{1}{n} \sum_{t=1}^{n}\left(\sigma_{1, t}^{2}+\alpha_{1, t}^{2}\right)\right)+h_{2}^{2}\left(\frac{1}{n} \sum_{t=1}^{n}\left(\sigma_{2, t}^{2}+\alpha_{2, t}^{2}\right)\right) \\
& +2 h_{1} h_{2}\left(\frac{1}{n} \sum_{t=1}^{n} \alpha_{1, t} \alpha_{2, t}\right)+1 \\
\stackrel{(a)}{\leq} & h_{1}^{2}\left(\frac{1}{n} \sum_{t=1}^{n}\left(\sigma_{1, t}^{2}+\alpha_{1, t}^{2}\right)\right)+h_{2}^{2}\left(\frac{1}{n} \sum_{t=1}^{n}\left(\sigma_{2, t}^{2}+\alpha_{2, t}^{2}\right)\right) \\
& +2 h_{1} h_{2}\left(\frac{1}{n} \sum_{t=1}^{n} \alpha_{1, t}^{2}\right)^{1 / 2}\left(\frac{1}{n} \sum_{t=1}^{n} \alpha_{2, t}^{2}\right)^{1 / 2}+1
\end{aligned}
$$

where (a) follows by Cauchy-Schwarz inequality.

By the concavity of the mutual information and applying Jensen's inequality [3] in bounds (38) in the limit when $n \rightarrow$ $\infty$, it can be shown that any achievable information-energy rate triplet $\left(R_{1}, R_{2}, B\right)$ should satisfy

$$
\begin{aligned}
R_{1} & \leqslant \frac{1}{2} \log _{2}\left(1+h_{1}^{2} \sigma_{1}^{2}\right), \\
R_{2} & \leqslant \frac{1}{2} \log _{2}\left(1+h_{2}^{2} \sigma_{2}^{2}\right), \\
R_{1}+R_{2} & \leqslant \frac{1}{2} \log _{2}\left(1+h_{1}^{2} \sigma_{1}^{2}+h_{2}^{2} \sigma_{2}^{2}\right), \\
B \quad & \leqslant 1+h_{1}^{2}\left(\sigma_{1}^{2}+\alpha_{1}^{2}\right)+h_{2}^{2}\left(\sigma_{2}^{2}+\alpha_{2}^{2}\right)+2 h_{1} h_{2}\left|\alpha_{1}\right|\left|\alpha_{2}\right|,
\end{aligned}
$$

for some $\sigma_{1}^{2}, \sigma_{2}^{2}, \alpha_{1}^{2}$, and $\alpha_{2}^{2}$ such that (37) is verified. Let $\mathcal{R}_{0}\left(\sigma_{1}^{2}, \sigma_{2}^{2}, \alpha_{1}^{2}, \alpha_{2}^{2}\right)$ denote this region. The capacity-energy region is contained within the union of all $\mathcal{R}_{0}\left(\sigma_{1}^{2}, \sigma_{2}^{2}, \alpha_{1}^{2}, \alpha_{2}^{2}\right)$ over all $0 \leqslant \sigma_{1}^{2}+\alpha_{1}^{2} \leqslant P_{1}$ and $0 \leqslant \sigma_{2}^{2}+\alpha_{2}^{2} \leqslant P_{2}$. In this union, it suffices to consider $\alpha_{1} \geqslant 0, \alpha_{2} \geqslant 0$, and $\sigma_{1}^{2}, \sigma_{2}^{2}$, $\alpha_{1}^{2}$, and $\alpha_{2}^{2}$ that saturate the input power constraint (i.e., (37) holds with equality). If $\beta_{i} \triangleq \frac{\sigma_{i}^{2}}{P_{i}} \in[0,1], i \in\{1,2\}$, such a region contains all information-energy rate triplets $\left(R_{1}, R_{2}, B\right)$ satisfying constraints (13) which completes the converse of the proof.

\section{REFERENCES}

[1] S. Bi, C. K. Ho, and R. Zhang, "Wireless powered communication: opportunities and challenges," IEEE Commun. Mag., vol. 53, pp. 117125, Apr. 2015.

[2] T. M. Cover, Some advances in broadcast channels. Academic Press, 1975, vol. 4, ch. 4.

[3] T. M. Cover and J. A. Thomas, Elements of Information Theory, 2nd Ed. Wiley, 2006.

[4] R. Durret, Probability Theory and Examples. New York, NY: Cambridge University Press, 2010.

[5] A. M. Fouladgar; and O. Simeone, "On the transfer of information and energy in multi-user systems," IEEE Communications Letters, vol. 16, no. 11, pp. 1733-1736, Nov. 2012.

[6] M. Gastpar, "Gaussian multiple-access channels under received-power constraints," in Proc. of IEEE ITW, 2004, pp. 452-457.

[7] P. Grover and A. Sahai, "Shannon meets Tesla: Wireless information and power transfer," in Proc. of IEEE ISIT, Jun. 2010, pp. 2363-2367.

[8] B. Gurakan, O. Ozel, J. Yang, and S.Ulukus, "Energy cooperation in energy harvesting wireless communications," in Proc. of IEEE ISIT, July 2012, pp. 965-969.

[9] I. Krikidis, S. Timotheou, S. Nikolaou, G. Zheng, D. W. K. Ng, and R. Schober, "Simultaneous wireless information and power transfer in modern communication systems," IEEE Comm. Mag., vol. 52, pp. 104110, Nov. 2014

[10] L. R. Varshney, "Transporting information and energy simultaneously," in Proc. of IEEE ISIT, Jul. 2008, pp. 1612-1616.

[11] A. D. Wyner, "Recent results in the Shannon theory," IEEE Trans. Inf. Theory, vol. 20, no. 1, pp. 2-10, 1974. 